\title{
Digital Solutions in Educators' Training: Concept for Implementing a Virtual Reality Simulator
}

\author{
Vlada V. Kugurakova ${ }^{1 *}$, Inna I. Golovanova ${ }^{1}$, Albina R. Shaidullina ${ }^{2}$, Elmira R. Khairullina ${ }^{3}$, \\ Natalia A. Orekhovskaya ${ }^{4}$ \\ ${ }^{1}$ Kazan (Volga region) Federal University, 420008, Kremlevskaya Street 18, Kazan, RUSSIA \\ ${ }^{2}$ Almetyevsk State Oil Institute, 423450, Lenin Street 2, Almetyevsk, RUSSIA \\ ${ }^{3}$ Kazan National Research Technological University, 420015, Karl Marks Street 68, Kazan, RUSSIA \\ ${ }^{4}$ Financial University under the Government of the Russian Federation, 125993, Leningradsky Prospekt 49, Moscow, RUSSIA
}

Received 2 April 2021 - Accepted 8 June 2021

\begin{abstract}
Over the past decade virtual reality technologies have been actively introduced into all spheres of life, affecting, among other things, the education sector. The purpose of this study is to develop a concept introducing a virtual reality simulator into educators' training. The underlying principle of the study is to design a virtual reality environment that, firstly, facilitates the development of conflict resolution competence for the students of pedagogical faculties, and secondly, facilitates its performance evaluation. In this article, approaches to design such a virtual reality simulator are discussed. The purpose of the simulator is to develop conflict resolution competences that are indispensable to future teachers. Besides the current study presents a set of situational cases, that are possible to resolve in a virtual reality environment. The resolution of those cases will help future teachers to prevent conflicts as well as to determine their behaviour in pre-conflict and conflict situations. The components of conflict resolution competence that are possible to develop with the help of the designed virtual reality simulator have been defined. The conducted study has also identified approaches to evaluate the effectiveness of conflict resolution competence development.
\end{abstract}

Keywords: virtual reality, pedagogy, conflict resolution competence, learning environment, immersive learning environment, educators' training, teachers' training, conflict, virtual agent, virtual reality technology

\section{INTRODUCTION}

Innovative solutions and scientific developments constantly open up new opportunities, allowing people to creatively approach the creation and transformation of familiar processes. Perhaps today humanity is in the midst of such changes, driven forward by virtual reality technologies (Akhmetgareyeva, 2020).

A few years ago, virtual reality was talked about in the future tense, it seemed inconceivable and it was associated only with programming, information technology and games.

In the last decade the applicability scope of virtual reality has significantly expanded. It is now used in the field of education. The results are very promising. Since students deal with a huge amount of information, it is important to ensure the best quality of comprehension, which to a great extent depends on the effectiveness of information delivery (Kvon et al., 2018; Levina et al., 2019). There is no other technology like virtual reality, which significantly improves and simplifies educational process. Virtual reality is used both to visualize the material being taught and to conduct laboratory studies and lessons. The examples are numerous.

However, an important aspect of education is active interaction with students, analysis of various acute conflict situations that require a quick and immediate solution. Young teachers, due to their inexperience and

(c) 2021 by the authors; licensee Modestum. This article is an open access article distributed under the terms and conditions of the Creative Commons Attribution License (http://creativecommons.org/licenses/by/4.0/). 


\section{Contribution to the literature}

- The concept of developing a VR simulator is presented in this paper. It contributes to the formation of future teachers' conflict resolution skills.

- The authors propose approaches, tools and algorithms for designing the immersive environment including multi-level situational tasks for typical conflict situations. The latest ones arise during the interaction of a student and a teacher.

- The empirical advantages of VR solutions show that VR and AR technologies contribute to the effective immersion students in a life situation and help them to find optimal pedagogical solutions.

lack of confidence, are often lost in such situations, having no idea of how to handle and solve the problem.

We decided to go beyond the familiar use of virtual reality and to develop a simulator for students of pedagogical specialities where they can train in an immersive environment and resolve conflict situations that often arise in the educational process.

\section{Virtual Reality in Education}

The use of virtual reality in education is becoming increasingly common, but pedagogy used in this environment is rarely explicit or claimed. Walker et al. (2020) review the current debate on virtual reality learning and explore the evolution of virtual reality environments in a pedagogical context. Kebritchi and Hirumi (2008). identified five different categories of typical learning theory: direct instruction, experiential learning, discovery learning, situated cognition and constructivist learning.

- Direct instruction. In the pedagogical model of direct instruction, skills and knowledge are acquired through teaching and reinforcement. Learning objectives are achieved through structured lessons accompanied by revision and reinforcement tutorials. This direct learning environment allows students to practice fundamental skills while reducing costs and working in a safe environment (Abramov et al., 2017).

- Experiential learning. Students are learning from their own experiences, whether in real life or in virtual settings. These experiences can be embedded in instances of thinking, seeing, feeling, handling and doing, in order to support learning process (Dewey, 1938). It is a form of active learning where a student participates in knowledge formation through his interaction with the environment (Santos \& Carvaho, 2013). This approach is particularly useful for building information modelling in virtual reality. By providing information in an experiential setting, on-site productivity can be improved and accidents reduced.

- Discovery learning. This teaching method is based on queries that arise when students who have undergone theoretical training, get new experience and use it to understand and explore new concepts. They are more likely to succeed in a discovery learning environment if they have necessary knowledge and engage in carefully planned learning activities (Roblyer et al., 1997).

- Situated cognition. The theory of situated cognition explains that knowledge is related to the physical, social and cultural context (Brown et al., 1989). This approach has been popularised in the work of Lave and Wenger (1991). and their theories of legitimate peripheral involvement. It may seem that all examples of experiential learning would be synonymous with forms of situated cognition. As the matter stands, scientific research has already some examples with structured learning opportunities in virtual reality environments.

- Constructivist learning. Social constructivist theories explain that learning does not simply occur through knowledge acquisition, but that knowledge is socially constructed by learners in response to stimuli (Vygotsky, 1978). Consequently, during constructivist learning learners use their past experiences to extract meaning from new knowledge and to construct individual understanding. Problem-solving ability and self-efficacy are just one of the examples that reveal the impact of self-esteem on learners' academic performance (Chi et al., 1989).

Using this categorisation, a pedagogical foundations' study of 35 recent virtual reality applications was conducted. It showed that the majority of these applications were used for experiential learning in the first place, and for discovery learning in the second place (Johnston et al., 2018). As one can see, virtual reality as a pedagogical tool is still underutilised. After gathering experience, learning continues through reflective observation, abstract conceptualisation and active experimentation (Kolb, 1984). In this context, a learning environment that uses only direct instruction does not provide learners with opportunities to use new knowledge and integrate it with existing understanding. It results in lost opportunities for teachers and students.

Pradhananga et al. (2020) describe the problem of training engineers who devote little or no time to communication skills during their professional training. 
Professionals must not only become experts in the technical aspects of their field, but also develop soft, i.e., communication skills in order to use their technical knowledge in an evolving, complex and globalised work environment. The research results provided an opportunity to assess the impact of non-traditional approaches - virtual reality approaches - on students' presentation skills. The results provide valuable feedback and insight into the implementation of alternative pedagogical teaching methods that in addition to technical knowledge integrate learners' developmental skills.

It is particularly relevant today to change the approach to the organisation of educators' training. In competence-based approach, special attention is paid to the practical component (Golovanova et al., 2019; Sidenko, 2019). What has to be considered is the ongoing under the influence of enhancing digital learning environment transformation of communicative functions that are significant in pedagogical interaction (Kirillova et al., 2020).

\section{Main Idea}

Our main motivation is to more actively use situational and constructivist learning paradigms in virtual reality, achieving a synergetic effect. A virtual reality headset takes a future teacher into a virtual classroom environment. He is given the opportunity to interact with virtual students through a variety of methods and environment interactive elements. Students interact with a teacher and environment. A key part of the whole simulation system is the emerging pedagogical situations, either initiated by a teacher, students or environment.

The concept of a user study is close to the methodology proposed by Barmaki and Hughes (2015), which offers training in demonstrating non-verbal cues. Non-verbal communication strategies are regularly noted in successful approaches to teachers' training (Mahon et al., 2010). The effects of such strategies as eye contact, prolonged gaze and proximity can have positive or negative effects on student behaviour and classroom management, depending on the situation and context (Laslett \& Smith, 1992). The other study (Lugrin et al., 2016) presents a system for skill training to cope with classroom misbehaviour.

\section{Expected Results}

Virtual reality simulator enables an authentic reconstruction of pedagogical process and various pedagogical situations that students will encounter in future teaching practise. In a virtual reality mistakes and errors are not grave. They serve as a means to elaborate behavioural strategy that will help to solve issues in a real educational process.
In traditional methodology, participants experience psychological barriers while acquiring communication skills. They are often uncomfortable and confused. However, this is not the case with virtual training (Kickmeier-Rust et al., 2020). Participants feel more relaxed and at ease. Virtual reality lessens their psychological pressure, while preserving educational benefits.

To summarize, implementing a virtual reality simulator into educators' training is expected to result in:

- First-hand experience participating in virtual conflict situations, that are based on the real cases;

- Development of diagnostic and reflexive skills that contribute to the organization of productive joint activities;

- Development of skills that help resolving conflicts in the educational process;

- Development of the ability to design, model and foresee your own behaviour and actions, taking into account the educational tasks that arise in complex conflict situations;

- Development of communicative culture, which is the basis for building interpersonal relationships;

- Development of quick decision-making and response in real conflict situations in professional pedagogical practice.

\section{Implementation of the Virtual Environment}

To extend training's immersive effect, students are transferred into a virtual reality of a regular classroom with standard physical dimensions $15 \mathrm{~m} \times 20 \mathrm{~m} \times 3 \mathrm{~m}$ (see Figure 1). It is equipped with double or single desks and can accommodate up to 30 people.

A pool of 30 different student characters has been developed. From this pool the system randomly generates a class of 10 students at the beginning of each session, providing a large number of possible class configurations. Each of the characters has an individual look so that a teacher can identify them by clothing, hairstyle, etc. Virtual pupils are just like real pupils. They have different appearance and belong to different ethnic groups and nationalities. The pool comprises as well as a small set of stereotypical personalities, for instance, fashionable ones, intellectual or sporty students, and 'nerdy' pupils.

The virtual characters were designed in software "Character Creator" (2021). To create a realistic animation IpiSoft Recorder (2021) and Mocap Studio (2021) were used. Later the outcomes were edited and applied to the student characters in programmes Autodesk (2021) and Maya (2021) before they were finally imported into the Unity (2021) game engine. The final number of triangles per character is between 22,000 - 25,000. Approximately 843,000 triangles were used for 


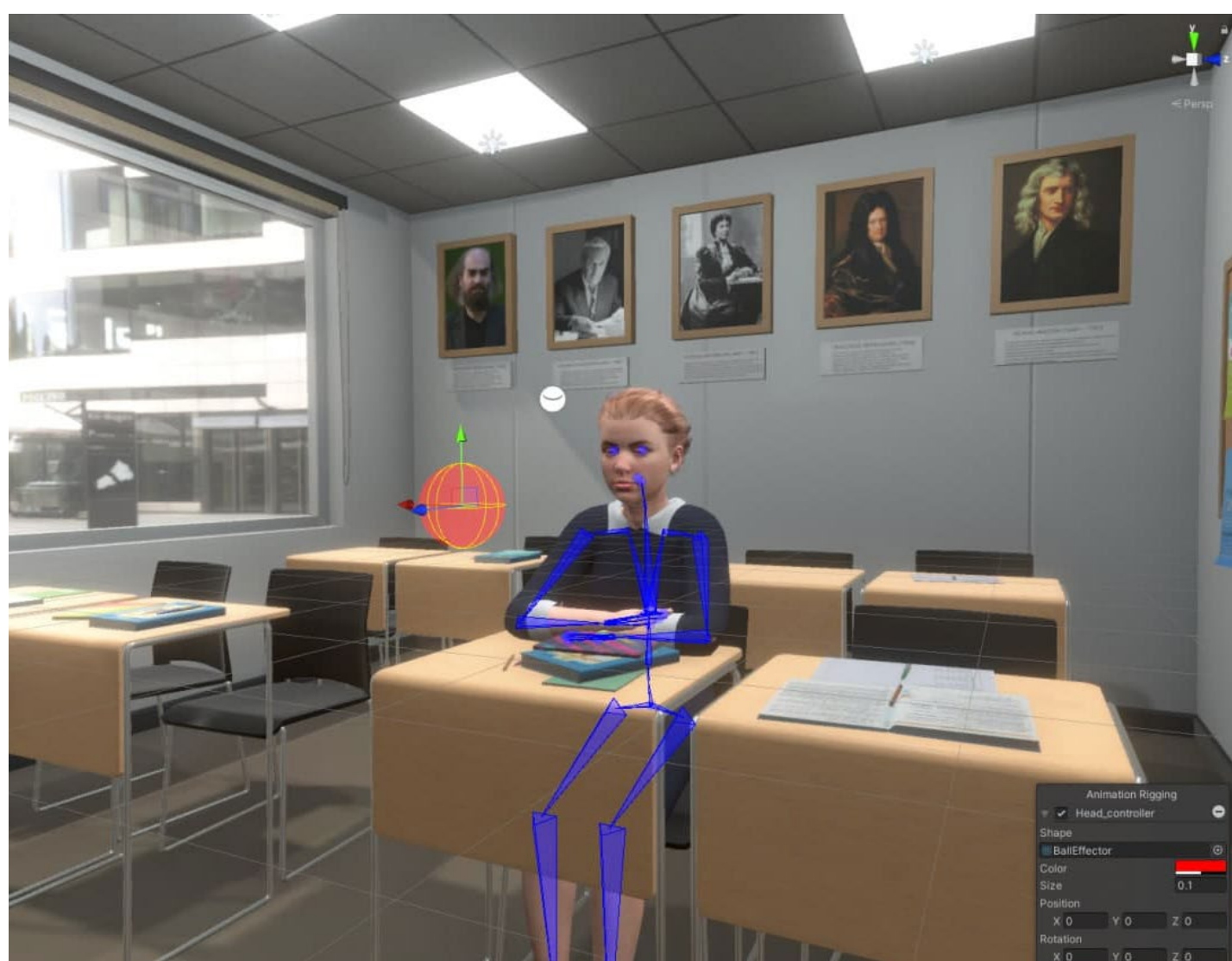

Figure 1. Virtual reality classroom

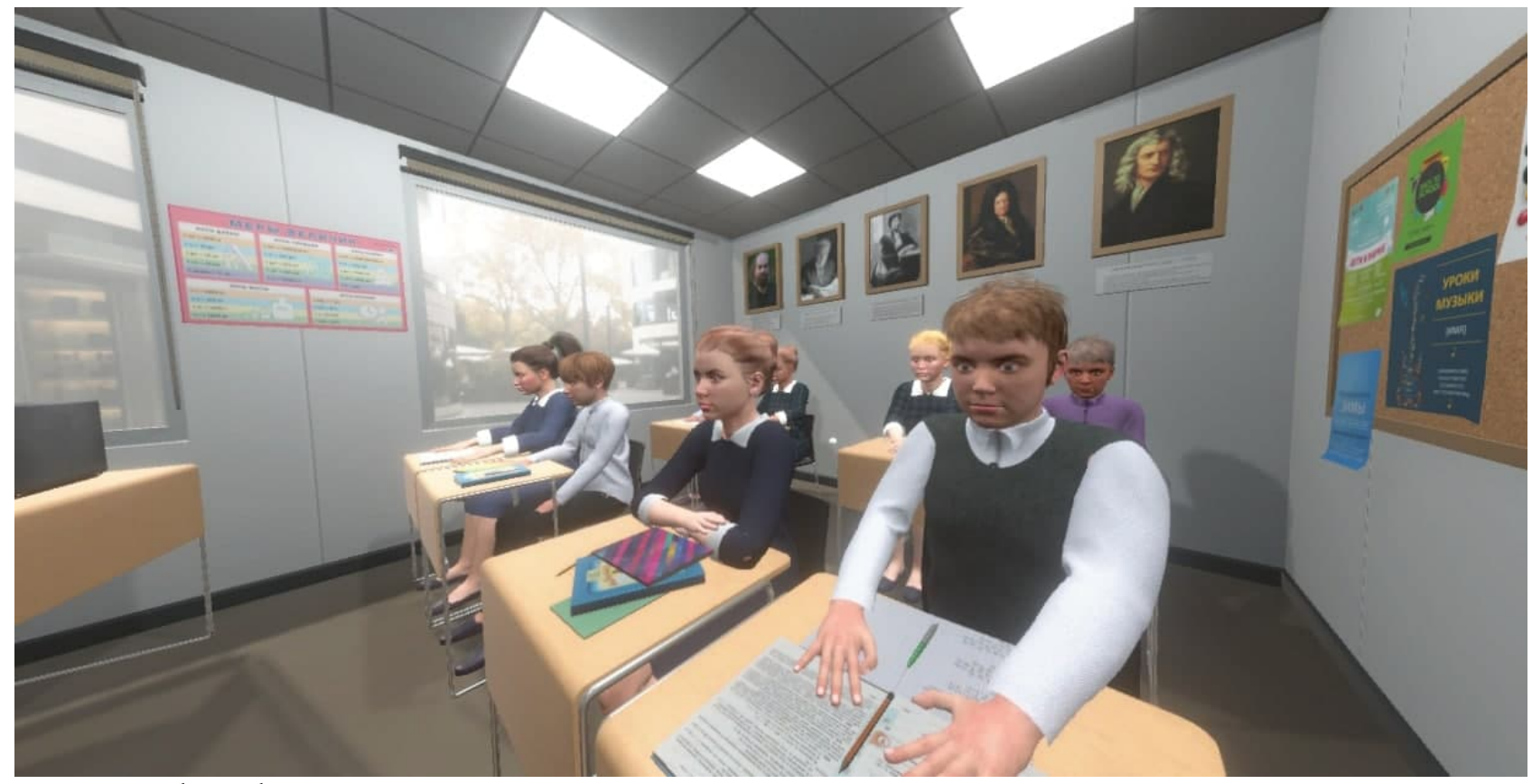

Figure 2. Virtual pupils

the entire environment, including furniture, walls and windows.

The virtual pupils (see Figure 2) look very conventional. It prevents users from full immersion into crisis management. To solve this issue it is planned to use another tool called MetaHuman Creator (2021) in the next iteration. With this tool it is possible to create a more realistic implementation of three-dimensional models of virtual students whose faces and facial expressions do not differ from those of real children. However, it means that a complete refactoring of the existing pilot solution for implementation on the Unreal Engine is needed (2021). 
The virtual environment includes some ambient sounds, e.g., recess bell and hallway noise.

Each virtual pupil is managed by a behaviour tree linked to the virtual world through individual perception and behaviour control modules. The main function of the behaviour tree is to define the active animation that is going to be played by the virtual pupil's finite animation automaton. This finite automaton handles the animations, their transitions and controls the corresponding sounds.

The perception module helps create a more plausible environment. It also adds to the dynamics of the scene by enabling virtual pupils' reaction to teacher's and fellow students' actions. The perception module basically mimics the humanoid senses of hearing and vision, which can be limited to realistic values. The module decides whether the perceiving pupil should react to a sound event or not.

System performance was evaluated with regards to average frame rate and end-to-end latency, perceived by a student in the teacher's role. This is an important technical element that allows to simulate a plausible environment for a teacher and to provide a safe and healthy environment that does not cause cyber sickness and provides an acceptable user experience.

Each of the pedagogical cases contains a verbal and visual introduction to the topic and study objectives. Visual and textual clues are used during teacher's virtual interactions with the class.

An educational psychologist participated in the simulator development. For each situational case he elaborated learning objectives, interaction methods, use of visual and verbal cues. Besides thanks to his participation the following changes were made to the immersive environment: a additional part of introductory content, some additional segments, different question wording, layout change, more interactive components. It was an iterative process, the psychologist interacted with the environment several times. Refining of the immersive environment has to be continued and all the necessary changes should be completed, so that the situational cases are perceived organically by a user as if they happen in a real classroom. To avoid cases' repetition the class composition and virtual characters are randomised. It enables new conflict situations and therefore new solutions.

\section{Pedagogical Situations}

Zhao and Ma (2020) examine situational learning of communication skills by simulating an interactive agent with multimodal feedback in a virtual reality. The virtual agent acts as a trainer and offers both verbal and nonverbal feedback to users. In a similar way, our virtual reality environment has standard and non-standard situations. Virtual pupils act as interactive agents, each of them has a set of reactions, actions and responses according to their psychological type.

It is essential to determine the components of conflict resolution competence that are to be trained and exercised in the virtual reality environment. Researchers have different opinions on this matter. For instance, according to Leonov (2011) conflict resolution competence includes teacher's conscious behaviour, forecasting of actions' results and consequences, social skills, courage, ability to experiment, critical thinking and so on. These are also part of cognitive, motivational and regulatory components. Conversely, Mityaeva (2012) identifies five components of conflict resolution competence, that are crucial for a future teacher in order to prevent conflict situations and to deal with them. These components are gnostic, regulatory or constructive, designing, reflexive-status, and normative. Mityaeva (2012) defines the main characteristics of each component and emphasizes that all of them are necessary in order to form a high level of conflict resolution competence.

Shcherbakova (2009) distinguishes cognitive, emotional, volitive, behavioural - practically effective components of conflict resolution competence and defines conditions and mechanisms of their formation in learning activities. To conclude, researchers share a common opinion that there is no unified approach both to the defined components of conflict resolution competence and to the methods and means of their formation and development.

To determine the objectives of the virtual reality simulator, the potential educational outcomes in the development of teacher's conflict resolution competence were evaluated, as well as the possibilities of pedagogical technologies used in an educators' training. More than 400 real cases of conflict interaction in educational process from practising teachers working at different levels of education were brought to the training platform for technological development of conflict scenarios. The cases were processed according to different parameters of their structuring.

Interpersonal conflicts with formal manifestation, with both objective and subjective causes, but only rational (in terms of influence) and single-factor (in terms of number of causes) were considered (see Figure 3). Multi-factor and irrational conflicts due to their complexity will be discussed in later studies.

Firstly, the situations were divided according to the participants of conflict interaction (see Figure 4): pupil pupil; teacher - teacher; subject teacher - pupil; homeroom teacher - pupil; parent - subject teacher; parent - homeroom teacher; parent - pupil; school administration - teacher; school administration - parent; school administration - pupil.

In the virtual reality simulator there are over 200 cases that cause the greatest difficulties for young teachers, i.e. the conflicts between a pupil and a teacher. 


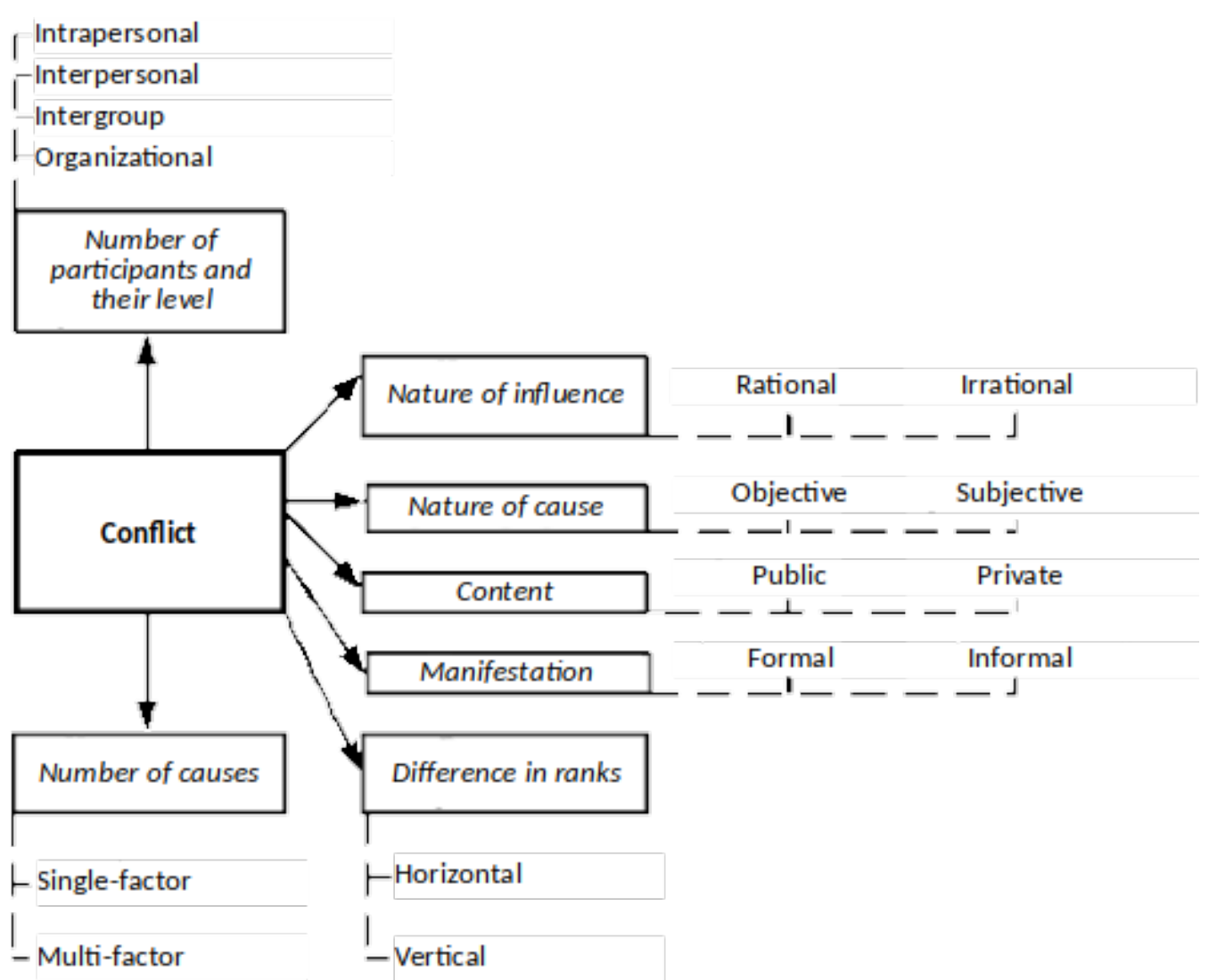

Figure 3. Main conflict types (Podoprigora, 2008)

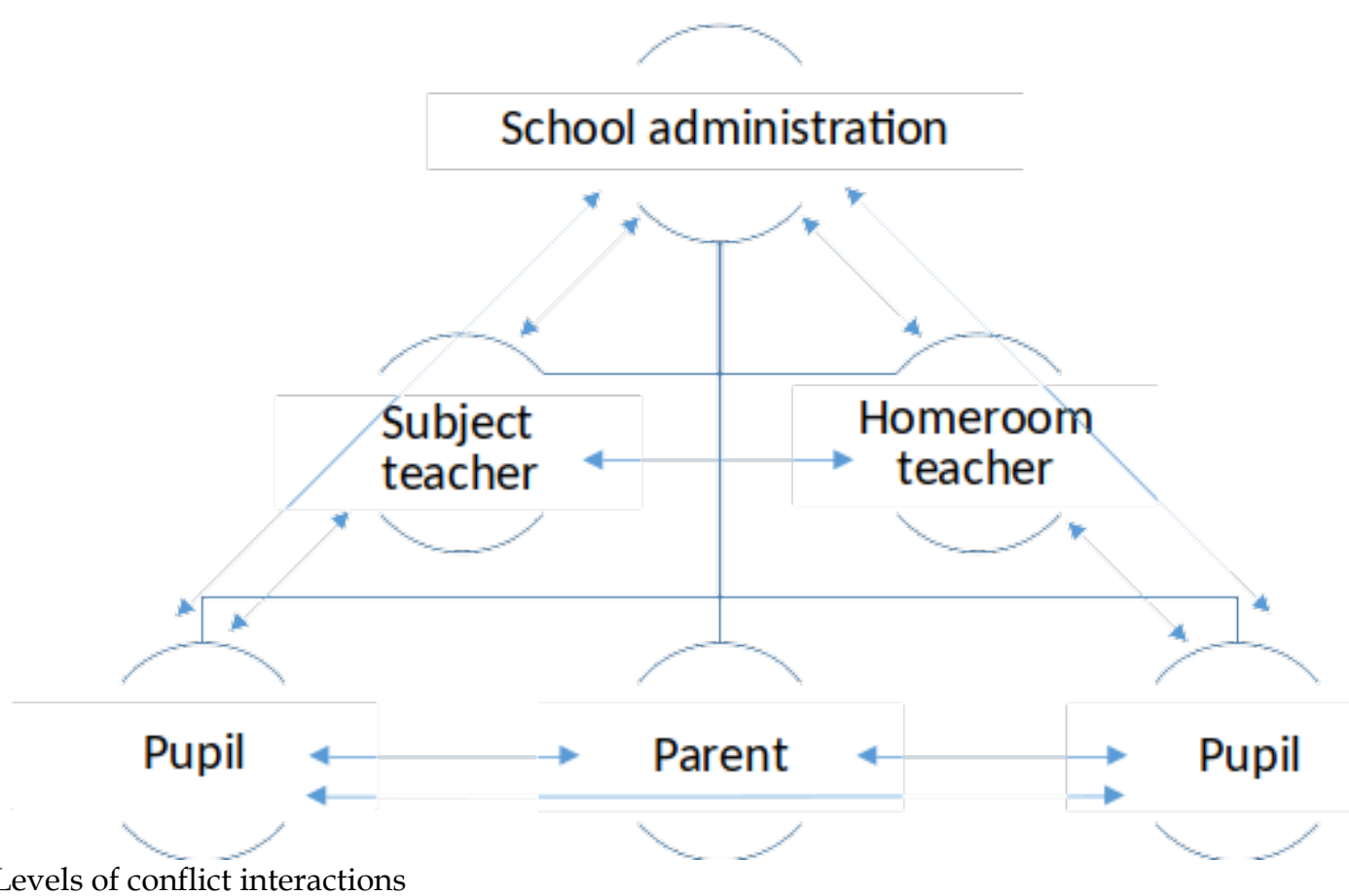

Figure 4. Levels of conflict interactions

These cases are divided into two groups according to the cause of a conflict: organisational-administrative, personal, and socio-psychological.

The organisational-administrative conflicts are those related to unfair evaluation of learning achievements, inconsistencies and contradictions in the goals of the teacher and individual students, non-compliance with rights and responsibilities of students, unequal treatment of students by the teacher, limited resources, contradictions between learning objectives and educational goals of students.

Cases related to differences in behaviour and life experiences, personality traits, teacher's lack of goodwill, lack of knowledge or motivation of the pupil, unfavourable physical conditions, uncertainty of 


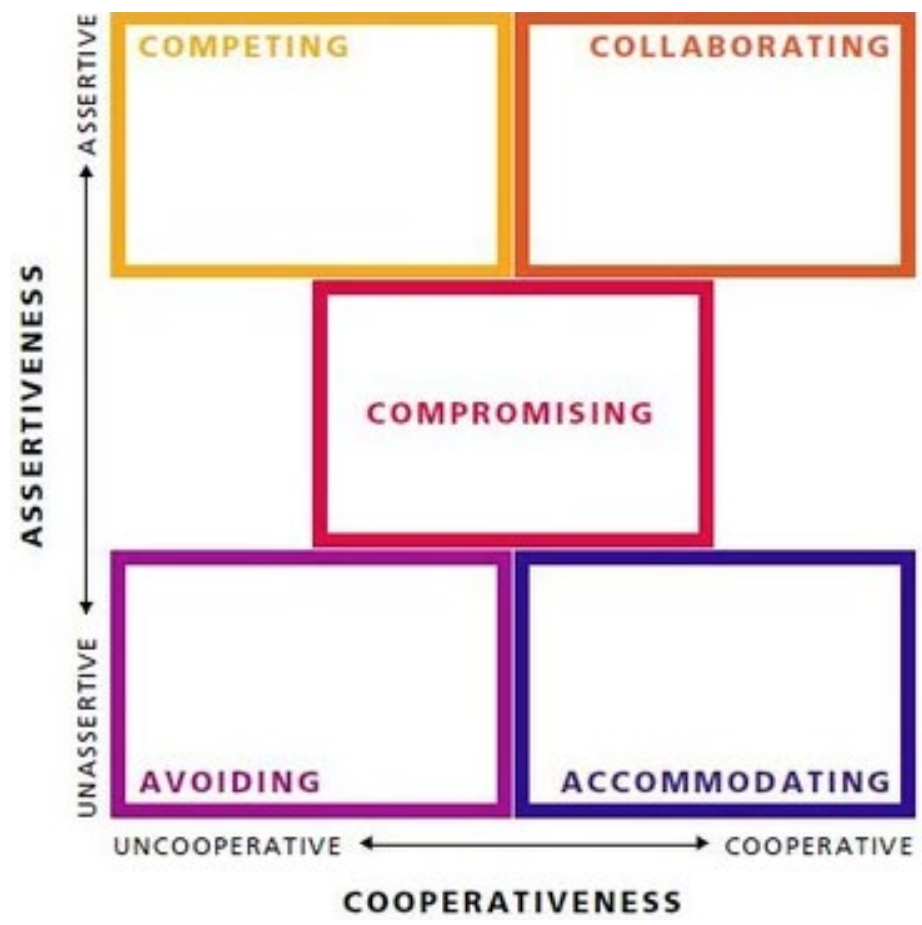

Figure 5. The Thomas Kilmann Model

learning prospects were considered to be personal and socio-psychological.

The teachers' reactions were developed on the basis of the Thomas Kilmann Model. According to it there are five behavioural strategies in a conflict situation: avoiding, accommodating, compromising, competing, and collaborating (see Figure 5).

In a virtual reality training, the user has to face a conflict situation and determine how critical it is for him and the pupil. He has to consider what he is ready to give up and how much he wants something in return. Based on the answers to these questions he should choose the reaction which he thinks would be the best in the given situation. Conflict, like any other process, has its developmental stages that are important to consider while planning a scenario: pre-conflict, open-ended conflict and post-conflict.

In the virtual reality simulator, the pre-conflict stage includes short introductory notes, which are based on such information as the stage of the lesson at which the situation unfolds, the pupil's age and corresponding crises that are relevant to it. This enables a more professional approach to the selection of behavioural strategies. The virtual reality simulator will have several levels of difficulty. At the entry level the user has to simply choose reactions and responses. At this level the user forms a behavioural strategy of reactions in typical situations. He has to assess only two parameters: the introductory information and the pupil's response. Thus a user learns to manage his emotions, to identify the object of the conflict and to choose the appropriate behavioural strategy and response.
At the next level there are more parameters to consider. For instance, there are various conflict personalities and therefore various response possibilities. The conflict personality typology that is used in the current study is suggested by psychologist J. G. Scott (1994). It includes:

- Type 1 "Steamroller" /"Sherman tank". People of this type demonstrate rude and aggressive behaviour, they are firmly convinced that they are always right, they demand from others immediate concessions.

- Type 2 "Hidden aggressor"/"Sniper". This type is associated with manipulation, instigation, and hidden aggression. The person of type 2 is convinced that justice must be restored by any means whatsoever and preferably by somebody else and not him.

- Type 3 "Angry child" /"Explosive person". These people are very emotional, but not angry in their nature. They are characterised by erratic emotional outbursts associated with situations of change in which they lose control and feel helpless.

- Type 4 "Complainant". People of this type are always discontent with everything what is going on around them. They put blame for it and for their own failures on other people.

- Type 5 "A quiet one". This person is reserved and secluded. He is very difficult to engage in conversation.

- Type 6 "Ultra-compliant". This type is difficult to recognise. One can characterize type 6 as 
accommodating and non-confrontational. People of this type let you down at any moment. Their words and actions are often at odds with each other.

At the entry level situation is encoded into each virtual pupil in the class, i.e., a user has to recognise the situation itself when choosing a pupil. At the second level, the situation is selected, whereas each virtual pupil represents a certain personality type, which a user has to recognise and in the course of the reaction choose the suggested tactics of behaviour that contribute to the resolution of conflict situations. Reaction chains consisting of 4-5 dialogue replicas were developed for a virtual reality training at this level.

The virtual reality simulator is being continuously improved. In future it is planned to include in the research team specialists from psychology and physiology. They will evaluate the effectiveness of a virtual reality technology not only in terms of teaching process organization, but also in terms of changes in the users' psycho-emotional state and physiological reactions in stressful situations (Kugurakova \& Ayazgulova, 2019)

\section{Study Design Process}

A pedagogical experiment was organised to assess the efficacy of a virtual reality training. Students of Master programmes undergoing "Training in pedagogical conflict resolution" were divided into two groups - a control group and an experimental one. In the control group, classes were conducted in accordance with the previously developed learning programme, which is structured as the alternation of theoretical minilectures and practical part with the help of various interactive teaching methods, such as discussions, games, etc. In the experimental group, in the practical part instead of such tasks as video analysis, discussions, or business games students had virtual reality exercises. For the assessment of course's mastering a diagnostic toolkit was developed, which focused both on the entrance diagnostics of conflict resolution knowledge and on the assessment of learning outcomes, conducted immediately after the training and within 3-4 weeks afterwards. The diagnostic toolkit included: a conflict knowledge assessment test; a questionnaire aimed at collecting information from students on their motivation and attitudes towards development of conflict resolution competence, use of virtual reality technologies in training and a rating chart on indicators and criteria for assessing development of components of conflict resolution competence.

The entrance diagnostics consisted of two parts. The first part contained a questionnaire. Its purpose was to collect background information on the age of the participants, their previous hands-on experience at school, their motivation to develop conflict management ability, self-assessment of their effectiveness level in dealing with conflict situations as well as about their gaming experience (i.e., how often they play video games) and their familiarity with virtual reality technology. The second part included testing. It was focused on the diagnostic function, which allowed to assess knowledge and level of primary skills of participants in the field of conflict management.

During training using VR-trainer, the students worked independently, while a teacher observed and organised reflection process. The control group was taught using classical teaching techniques. After completing the training, students in both experimental and control groups were offered a knowledge test and a control task, where an expert assessment of skill development in conflict management was used. In addition, students in the experimental group completed a questionnaire to evaluate their training and to evaluate the recommendations given during the training. They were also asked to compare the virtual reality training with traditional learning techniques. Three to four weeks after the training, participants were asked to complete a final test, called the retention test, to assess their level of knowledge retention and the level of self-efficacy.

\section{DISCUSSION AND CONCLUSION}

The results of the approach comparison showed that the vast majority of participants who tried virtual reality technology had a very positive experience. In fact, more than $75 \%$ of the sample gave a score above one on all three statements, comparing virtual reality learning with traditional learning.

The results thus prove the effectiveness of virtual reality training for both knowledge acquisition and knowledge retention. This result is a further support of the Månsson's (2018) findings, who demonstrated the effectiveness of virtual reality pre-learning prior to reallife experiences.

The introduction of virtual reality technologies can be a reliable tool in shaping conflict resolution skills of future teachers.

In the course of this study some typical conflict situations were analysed and a pool of situational tasks was compiled. Moreover, a virtual reality simulator prototype was developed, and its effectiveness was evaluated. At the moment, the project is being tested on a control group of students of pedagogical faculties. After that, the virtual reality simulator will be integrated into educators' training.

In conclusion, a virtual reality learning method is a suitable way of vocational education and training, even though these technologies are yet more expensive than traditional teaching methods. An important finding is that learning using virtual reality is perceived in a more concise and focused way, in contrast to lengthy full-day seminars covering the same scope of material. In the 
medium and long term, virtual reality and augmented reality will become a prominent part of the educational horizon. With the current study, some empirical evidence on the benefits of virtual reality solutions could be introduced. However, a clear limitation is the narrow degree of freedom in the presented scenarios. Furthermore, the relatively small number of participants is a constraint in results' interpretation.

As with the most educational activities, the question of transferability of training effects into the real world remains open.

Author contributions: All authors have sufficiently contributed to the study, and agreed with the results and conclusions.

Funding: This paper has been supported by the Kazan Federal University Strategic Academic Leadership Program.

Declaration of interest: No conflict of interest is declared by authors.

\section{REFERENCES}

Abramov, V., Kugurakova, V., Rizvanov, A., Abramsky, M., Manakhov, N., Evstafiev, M., \& Ivanov, D. (2017). Virtual Biotechnological Lab Development. BioNanoScience, 7(2), 363-365. https://doi.org/ 10.1007 / s12668-016-0368-9

Akhmetgareyeva, R.K. (2020). Pedagogical problems of training line engineers with a creative thinking style. Vestnik NCBŽD, 1(43), 5-9.

AutoDesk. (2021). https:/ / www.autodesk.ru

Barmaki, R., \& Hughes, C. E. (2015). Providing real-time feedback for student teachers in a virtual rehearsal environment. Proceedings of the 2015 ACM on International Conference on Multimodal Interaction, ICMI '15 (pp. 531-537). ACM. https:/ / doi.org/10.1145/2818346.2830604

Brown, J. S., Collins, A., \& Duguid, S. (1989). Situated cognition and the culture of learning. Educational Researcher, 18(1), 32-42. https://doi.org/10.3102/ 0013189X018001032

Character Creator. (2021). Reallusion. https:/ / www.reallusion.com/character-creator/

Chi, M. T., Bassok, M., Lewis, M., Reimann, P., \& Glaser, R. (1989). Self-explanations: how students study and use examples in learning to solve problems. Cognitive Science, 13(2), 145-182. https://doi.org/ $10.1207 /$ s15516709cog1302_1

Dewey, J. (1938). Experience and education. Simon and Schuster.

Golovanova, I. I., Telegina, N. V., \& Donetskaya, O. I. (2019). Teacher training on the basis of developed system of competence formation assessment. Education and self-development, 1, 58-68.

IpiSoft Recorder. (2021). https://www.ipisoft.com/download/

IpiSoft.

Johnston, E., Olivas, G., Steele, P., Smith, C., \& Bailey, L. (2018). Exploring pedagogical foundations of existing virtual reality educational applications: a content analysis study. Journal of Educational Technology Systems, 46(4), 414-439. https:/ / doi.org/ $10.1177 / 0047239517745560$

Kebritchi, M., \& Hirumi, A. (2008). Examining the pedagogical foundations of modern educational computer games. Computers and Education, 51, 1729. 1743.

https:/ / doi.org/10.1016/j.compedu.2008.05.004

Kickmeier-Rust, M. D., Leitner, M., \& Hann, P. (2020). Virtual reality in professional training: An example from the field of bank counselling. 2020 6th International Conference of the Immersive Learning Research Network (pp. 210-214). California Polytechnic State University. https://doi.org/ 10.23919/iLRN47897.2020.9155083

Kirillova, G. I., Grunis, M. L., Levina, E. U., \& Golovanova, I. I. (2020). Actualisation of the teacher's communication competence in digital activities. Kazan Pedagogical Journal, 4(141), 38-45.

Kolb, D. A. (1984). Experiential learning: Experience as the source of learning and development. Prentice Hall.

Kugurakova, V., \& Ayazgulova, K. (2019). Neurotransmitters level detection based on human bio-signals, measured in virtual environments. Advances in Intelligent Systems and Computing, 848, 209-216. https:/ / doi.org/10.1007/978-3-319-993164_28

Kvon, G. M., Vaks, V. B., Masalimova, A. R., Kryukova, N. I., Rod, Y. S., Shagieva, R. V., \& Khudzhatov, M. B. (2018). Risk in implementing new electronic management systems at universities. Eurasia Journal of Mathematics, Science and Technology Education, 14(3), 891-902. https://doi.org/10.12973 / ejmste/81060

Laslett, R., \& Smith, C. (1992). Effective classroom management: a teacher's guide. Routledge.

Lave, J., \& Wenger, E. (1991). Situated learning: Legitimate peripheral participation. Cambridge University Press. https:/ / doi.org/10.1017/CBO9780511815355

Leonov, N. I. (2021). Educator's conflict resolution competence - Education psychology in the XXI century: theory and practice - 2011. https://psyjournals.ru/ education21/issue/55838_full.shtml

Levina, I. D., Ukolova, L. I., Lavrentyeva, E. Y., Akhilgova, M. T., Zharikov, Y. S., Popova, O. V., Semyanov, E. V., Malanov, I. A., Muskhanova, I. V., Magomeddibirova, Z. A., Bazaeva, F. U., \& Isaeva M. A. (2019). Nursing home conditions for elderly people and its peculiarities of their adaptation. EurAsian Journal of BioSciences, 13(2), 1549-1555.

Lugrin, J.-L., Latoschik, M. E., Habel, M., Roth, D., Seufert, C., \& Grafe, S. (2016). Breaking bad behaviors: A new tool for learning classroom management using virtual reality. Frontiers in ICT, 
3(26), 1-21. https://doi.org/10.3389/fict.2016. 00026

Mahon, J., Bryant, B., Brown, B., \& Kim, M. (2010). Using second life to enhance classroom management practice in teacher education. Educational Media International, 47(2), 121-134. https://doi.org/ 10.1080/09523987.2010.492677

Månsson, J. (2018). Using a virtual fire extinguisher as a tool for safety training (Master thesis). Lund University.

Maya. (2021). https://www.autodesk.ru/pr-oducts/ maya

MetaHuman Creator. (2021). https://www.unrealengine.com/en-US/metahuman-creator

Mityaeva, A. M. (2012). Conflict resolution competence of the future professional as a subject of scientific research. Scholarly notes OGU. Series: Humanities and social sciences, 2, 273-280. https://cyberleninka.ru/ article/n/konfliktologicheskaya-kompetentnostbuduschego-spetsialista-kak-predmet-nauchnogoissledovaniya

Mocap Studio. (2021). https://www.rokok-o.com/ studio

Podoprigora, M.G. (2008). Organisational behavior. Study guide for undergraduate and graduate students. Publishing house TTI UFU.

Pradhananga, P., ElZomor, M., Santi, G., \& Zhang, L. (2020, June). Fostering virtual reality environments to advance construction and engineering students' interpersonal skills [Paper presentation]. 2020 ASEE Virtual Annual Conference Content Access, Virtual On line.

Roblyer, E., Edwards, J., \& Havriluk, M. A. (1997). Integrating educational technology into teaching. Merrill.
Santos, L., \& Carvaho, C. V. (2013). Improving experiential learning with haptic experimentation. International Journal of Online Engineering, 8, 7-9. https:// doi.org/10.3991/ijoe.v9iS8.3309

Scott, J. G. (1994). The empowered mind: How to harness the creative force within tou. VIS.

Shcherbakova, O. I. (2009). Formation and development of the professional's conflictological culture in context learning. Psychopedagogy in LawEnforcement Authorities, 3, 15-19. https://cyber leninka.ru/article/n/formirovanie-i-razvitiekonfliktologicheskoy-kultury-spetsialista-vkontekstnom-obuchenii

Sidenko, E. A. (2019). Innovative activity of teachers as a result of project management realization in the educational institution. Vestnik NCBŽD, 3(41), 7180.

Unity. (2021). https:/ / unity3d.com

Unreal Engine. (2021). https:/ / www.unr-ealengine.com Vygotsky, L. (1978). Mind in society. Harvard University Press.

Walker, J., Towey, D., Pike, M., Kapogiannis, G., Elamin, A., \& Wei, R. (2020). Developing a pedagogical photoreal virtual environment to teach civil engineering. Interactive Technology and Smart Education, 17(3), 303-321. https:/ / doi.org/10.1108/ ITSE-10-2019-0069

Zhao, Z., \& Ma, X. (2020). Situated learning of soft skills with an interactive agent in virtual reality via multimodal feedback. Adjunct Publication of the 33rd Annual ACM Symposium on User Interface Software and Technology (pp. 25-27). Association for Computing Machinery New York NY United States.

\section{http://www.ejmste.com}

